\title{
LA PATOLOGÍA UROLÓGICA EN LA OBRA DE ABULCASIS
}

Ignacio Otero Tejero, Alvaro Serrano Pascual, Javier Chicharro Almarza, Jesús Mํa Golbano Ablanque, Fernando Leal Hernández, Bernabé Pozo Mengual, Carlos Merino Hernáez, Pilar González Peramato Gutiérrez', Antonio Escolano Chamoix, y Manuel Sanz Redondo.

Servicio de Urología y Servicio de Anatomía Patológica'. Hospital Universitario de Guadalajara. Guadalajara. España.

Resumen.- OBJETIVO: Realizar un estudio de la obra quirúrgica de Abulcasis en el campo de la Urología, conocer el instrumental y las diferentes técnicas empleadas así como las innovaciones introducidas para solucionar algunos aspectos de la patología urológica.

MÉTODOS: Se ha revisado y traducido del latín la parte urológica del libro XXX del Tasrif utilizando la edición editada en Estrasburgo en 1532.

RESULTADOS: El autor apenas se refiere a los aspectos clínicos de las diferentes patologías del aparato genitourinario centrándose en el tratamiento quirúrgico de las mismas. Utiliza con asiduidad el cauterio sobre todo para controlar la hemorragia en las diferentes intervenciones. Se puede comprobar que algunos pasos quirúrgicos se continúan practicando en la actualidad.
Ignacio J. Otero Tejero Servicio de Urología Hospital Universitario de Guadalajara Donantes de sangre, $s / n$ 19002 Guadalajara. (España).

ijoteo $1 @ y a h o o . e s$
CONCLUSIONES: Abulcasis es el primer médico nacido en España que estudia el tratamiento quirúrgico de la patología urológica y que hace un estudio gráfico del instrumental utilizado. Aporta innovaciones técnicas en diversas intervenciones y describe por primera vez la talla vesical en la mujer, la litotricia vesical y uretral.

Palabras clave: Técnicas quirúrgicas. Cirugía urológica. Historia de la Urología.

Summary.- OBJECTIVES: To perform a study on the surgical work of Abulcasis in the field of urology, to know the surgical tools and different techniques used, as well as the innovations applied to solve some features of urological diseases.

METHODS: The part on urology from the book XXX by Tasrif, the edition from Strasbourg in 1532 was reviewed and translated from Latin.

RESULTS: The author does not refer much to the clinical features of the various pathologies of the genitourinary apparatus; he focuses instead on the surgical treatment of them. The author usually used the "cautery" mainly to control hemorrhage in various operations. Some of these surgical practices are being used nowadays.

CONCLUSIONS: Abulcasis was the first doctor born in Spain that studied the surgical treatment of urological diseases and performed a graphic study on the tools used. He introduced technical innovations on different operations and described for the first time the vesical lithotomy on women, and vesical and urethral lithotripsy.

Keywords: Surgical techniques. Urological surgery. History of Urology. 


\section{INTRODUCCIÓN}

La patología urológica se ha conocido desde la antigüedad. Las noticias que tenemos sobre el enfoque etiopatogénico, diagnóstico y terapéutico de las diferentes afecciones del aparato genitourinario en España son escasas en la Edad Antigua, apareciendo las primeras obras escritas durante la invasión árabe de la Península Ibérica.

La ocupación árabe de España influyó de manera importante en el desarrollo científico de nuestro país. La Medicina en aquella época tenía una gran influencia de los autores clásicos, aunque empezaron a despuntar los médicos musulmanes Rhazes y Avicena que influyeron de una manera muy destacada hasta el seiscientos.

El siglo más importante de la Medicina árabe española es el XII, en el que aparecen las figuras de Avenzoar, Averroes y Maimónides, siendo éste último de origen judío. En los siglos anteriores destacaron Ibn Habib, Abulcasis, Ibn Yulyul, Arid Ben Said El Kateb, Abenguefit y Abu I-Ala Zuhr. Sin duda alguna y desde el punto de vista de nuestra especialidad nuestro interés lo debemos centrar en Abulcasis por ser el primer cirujano nacido en la Península lbérica que cita y describe la resolución quirúrgica de algunas afecciones del aparato genitourinario.

Abu-I-Qasim Khalaf ibn Abbas Al-Zahrawi nació entre los años 936 y 940 en Medina Zahara, ciudad que en la actualidad se encuentra en ruinas, muy cerca de Córdoba. Murió en su ciudad natal en el año $1013(6,10)$.

Se le conoce por muchos nombres: Abulcasis, Albucasis, Alcarani, Alsarani, Aicaravi, Alcaravius, Alsaharavius, Al-Zahrawi, Buchasis, Bulcasim, Bulcasis, Bulcari, Calaf, Chalaf, Ezzahrawi, Jalaf, Schalaf, Zahravius $(10,12)$.

Su padre llamado Abbas, cuya familia procedía de Arabia Saudí, fue médico y Al-Zahrawi aprendió de él su oficio, posiblemente y entre otros motivos, para continuar con su clientela (2).

Hay datos no concluyentes que nos dicen que Abulcasis se trasladó a Bagdad para completar sus conocimientos de medicina (10), siendo ésta una costumbre frecuente entre los estudiantes musulmanes, ya que en esta ciudad estaba localizado, junto con Damasco, uno de los hospitales más importantes del Islam (11). Posteriormente desarrolló su trabajo en Córdoba y Medina Zahara.
De profundas creencias religiosas no había entre sus pacientes diferencias de clase social ni de religión. Abulcasis atendió a cristianos heridos en las batallas de la época, citándose la intervención que practicó con éxito a uno de ellos para extraer una flecha del cuello (10).

Fue médico de la corte de los califas Omeyas Abd-Er-Rahman III y de su hijo al-Hakam II al-Mustanir. Sin duda este califato corresponde con el periodo de máximo esplendor de la ocupación musulmana de la Península lbérica. Córdoba destacó no solo en el aspecto militar y político sino en el campo de las ciencias teniendo un gran renombre por sus numerosas bibliotecas y escuelas donde se enseñaban materias de todo tipo.

La gran Escuela de Medicina de Córdoba competía en aquella época con la Escuela de Salerno que posteriormente fue la precursora de muchas facultades de Medicina de Europa. Abulcasis enseñaba sus conocimientos a diversos alumnos a los que admitió con él en su práctica médica, como se puede deducir en su tratado de cirugía al dirigirse a un posible auditorio como "sus hijos", y en un grabado del manuscrito encontrado en Lieja titulado "La higiene de Abulcasis", en la que aparece bajo la figura de un profesor (10).

Escribió un libro titulado Kitab al-tasrif li-man ayiza 'an al-ta'lif, más conocido momo "Kitab al-tasrif", "Altasrif" o "Tasrif", en el que reúne todos sus conocimientos. El título quiere decir "Libro de la disposición médica para aquellos que no son capaces de saberlo por sí mismos" (7) aunque también se le conoce con los nombres de "La receta" o "La prácti$\mathrm{ca}^{\prime}$. Se divide en treinta libros o "maqalas", quince de "Practica" y quince de "Teórica" (30), tratando los temas quirúrgicos de las diferentes especialidades en el libro XXX (27).

La parte quirúrgica de esta obra ha sido considerada, sin ningún género de dudas, uno de los principales textos dedicados a la Cirugía de la Edad Media y por la que el autor alcanza un lugar importante en la Historia de la Medicina (14). La Escuela de Traductores de Toledo se encargó de traducirla del árabe al latín en el siglo XII, junto con obras tan emblemáticas como el "Canon" de Avicena y el "Liber Almansorem" de Rhazes. El equipo traductor estaba formado entre otros por Daniel de Morley, Roberto Chester, Alfredo Angélico, Marcos de Toledo y dirigido por Gerardo de Cremona (21).

No existen muchas ediciones de la obra completa del "Tasrif", pero de la parte quirúrgica se han hecho varias en el Renacimiento como son las editadas en Venecia, Estrasburgo y Basilea. 
Abulcasis tuvo una gran influencia desde la Edad Media hasta el siglo XIX (1). La importancia que tuvo en el desarrollo de la Cirugía en general y la Urología en particular se puede comprobar en las citas que hacen de sus textos cirujanos tan importantes como Guy de Chauliac y Pietro Argellata diciendo este último que Abulcasis "es sin duda el jefe de todos los cirujanos" (4). El autor latino Pablo Ricio alaba a nuestro cirujano con estas palabras "No me avergüenza decir, con perdón de los demás, que Abulcasis no es inferior a ningún médico después del padre de la Medicina, Hipócrates, y su intérprete, Galeno" (12).

La aparición en el siglo $X$ de la figura de Abulcasis representa sin ningún género de dudas el comienzo del desarrollo de la Urología en España. La descripción de las diferentes técnicas quirúrgicas y del instrumental utilizado así como las innovaciones introducidas para solucionar algunos aspectos de la patología urológica aumentan el interés en estudiar la obra de este médico cordobés.

\section{MATERIAL Y MÉTODOS}

Se ha procedido a traducir del latín la parte que corresponde a la patología urológica del libro XXX del Tasrif. Para ello se ha escogido el libro de Teodoro Prisciano en el que se recopilan las obras de Octavio Horatiani "Octavio Horatiani rerum Medicarum Lib. Quatuor" y la parte correspondiente al tratado de cirugía de Abulcasis "Albucasis Chirurgicorum omniu Primarii, Lib tres". Fue editada en Estrasburgo en el año 1532 por loannem Schottum (26). Los textos y las imágenes se han obtenido en la Biblioteca Digital Dioscórides, Biblioteca Histórica "Marqués de Valdeci"la", de la Universidad Complutense de Madrid.

\section{Los capítulos traducidos son los siguientes:}

- Capítulo 39. "Del modo de cauterizar los riñones".

- Capítulo 40. "Del modo de cauterizar la vejiga".

- Capítulo 55. "Sobre la curación de los niños que nacen con los lugares de la orina no perforados".

- Capítulo 56. "Sobre el "Albather" que ocurre en el prepucio y en la punta del pene y sobre la corrupción, la negrura y la adherencia".

- Capítulo 57. "Acerca de la limpieza y curación de los niños de algo que no tienen bien".

- Capítulo 58. "A cerca de la extracción de la orina retenida en la vejiga".

- Capítulo 59. "De la forma de "clisterización" de la vejiga con la clepshydra".

- Capítulo 60. "De la extracción de la piedra".

- Capítulo 61. "De la extracción de la piedra de las mujeres".

- Capítulo 62. "Del corte (sección) sobre la hernia acuosa".

- Capítulo 63. "De la curación de la rama larga carnosa".

- Capítulo 64. "De la curación de la rama larga que existe con la vid".

- Capítulo 68. "Del ablandamiento de la piel de los testículos".

- Capítulo 69. "De la castración".

- Capítulo 70. "De la curación de las dos formas del hermafroditismo".

\section{RESULTADOS Y COMENTARIOS}

El tratado de Cirugía de Abulcasis tiene como base la obra quirúrgica de Pablo de Egina además de los diferentes tratados de los clásicos que tanto influyeron en los médicos de la Edad Media (23). A estos textos nuestro autor aporta sus ideas y su experiencia personal así como el diseño de su propio instrumental quirúrgico.

La primera parte del tratado de cirugía está dedicado a la cura mediante el cauterio. La llamada

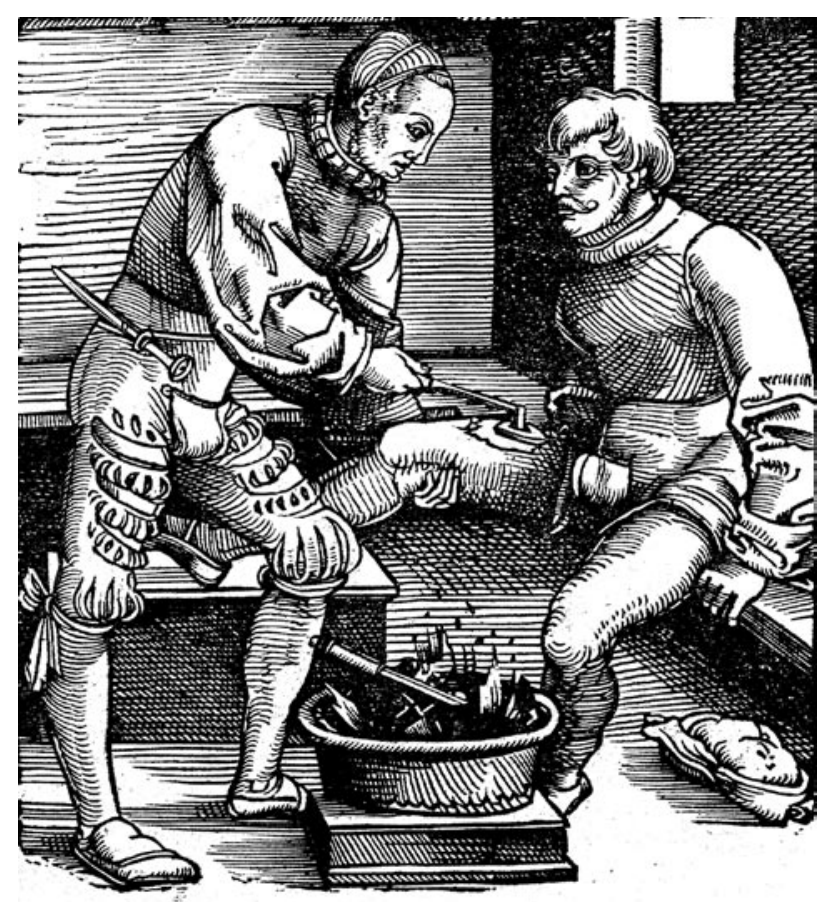

FIGURA 1. Práctica del cauterio

Tomado de Teodoro Prisciano "Albucasis Chirurgicorum omnium Primarii, Lib tres". 1532 por loannem Schottum. Biblioteca digital Dioscórides. Biblioteca Marqués de Valdecilla. Universidad Complutense. Madrid. 
"técnica de las ustiones" ha sido citada por los clásicos y fue muy utilizada por la medicina árabe (13).

Se han encontrado referencias al tratamiento de la patología urológica en la parte dedicada a la utilización de cauterio (Figura 1). La cauterización ha sido un método muy utilizado y recomendado por Abulcasis no solo en el quehacer quirúrgico sino para ser aplicado en el tratamiento de algunas afecciones como la gota (5). El dolor en la zona lumbar, no se refiere al cólico renoureteral, y la incontinencia urinaria han sido tratados mediante la aplicación del cauterio, apuntando el lugar exacto donde debe de ser aplicado en la región lumbar y en el hipogastrio "parte inferior de la fertilidad", aunque para estas dos situaciones en diversas partes de su obra recomienda tratamientos farmacológicos.

El cauterio lo va a utilizar en cirugía para ayudar a prevenir y manejar con más seguridad las hemorragias que se producen en el curso de una intervención quirúrgica y para tratar de manera correcta

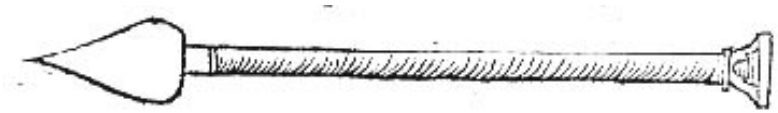

FIGURA 2. Spatumile subtili.

Tomado de Teodoro Prisciano "Albucasis Chirurgicorum omnium Primarii, Lib tres". 1532 por loannem Schottum. Biblioteca digital Dioscórides. Biblioteca Marqués de Valdecilla. Universidad Complutense. Madrid.

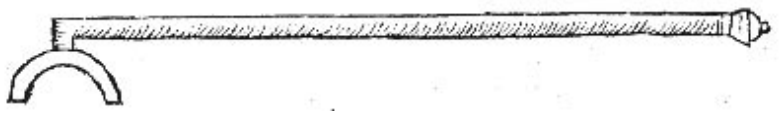

FIGURA 3. Cauterio lunar

Tomado de Teodoro Prisciano "Albucasis Chirurgicorum omnium Primarii, Lib tres". 1532 por loannem Schottum. Biblioteca digital Dioscórides. Biblioteca Marqués de Valdecilla. Universidad Complutense. Madrid.

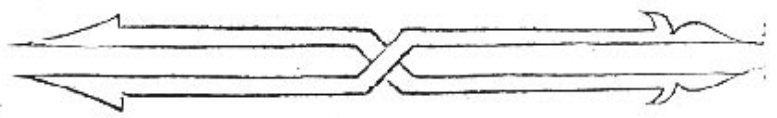

FIGURA 4. Tijeras

Tomado de Teodoro Prisciano "Albucasis Chirurgicorum omnium Primarii, Lib tres". 1532 por loannem Schottum. Biblioteca digital Dioscórides. Biblioteca Marqués de Valdecilla. Universidad Complutense. Madrid. una herida o lesión que está infectada (corrompida). Con el objeto de practicar la cauterización propone una serie de instrumentos diseñados para aplicar el calor en las diversas partes del organismo según la afección que esté tratando.

Uno de los aspectos más llamativos de la parte quirúrgica del Tasrif son los grabados del instrumental quirúrgico recomendados por el autor. Es el primer libro que incluye ilustraciones de esta naturaleza (8). Hay una gran diferencia con los grabados del libro original y algunos de éstos, como se puede observar, tienen una baja calidad descriptiva.

\section{La patología urológica citada es la siguiente:}

\section{1) Imperforación uretral. Estenosis congénita de mea- to uretral.}

Define esta patología de esta manera: "la punta de su miembro viril no está perforada o la apertura es pequeña".

En el caso de que exista una imperforación o una estenosis importante, practica una incisión en la zona del meato mediante el "spatumile subtili" (Figura 2), seguido de la colocación de un clavo de plomo a modo de tutor. Si la estenosis no es importante recomienda realizar una dilatación con un clavo de plomo durante varios días retirándolo únicamente para que el niño pueda orinar (25).

\section{2) Hipospadias.}

La referencia a esta patología es clara: "la apertura (meato uretral) no está en su lugar", "la apertura la tienen junto al final de la punta del pene", "no pueden orinar hacia delante", "elevan el pene con la mano hacia arriba" y "no tienen descendencia porque el esperma no llega a la matriz correctamente". Solamente la define y no propone ningún tratamiento incluso para aquellos que no pueden tener hijos debido a que eyaculan fuera de la vagina (25).

\section{3) Lesiones inflamatorias en el prepucio y el glande. "Albather".}

El término "albather" $(28,29)$ se aplica en general a las lesiones inflamatorias y tumorales del pene, pudiendo englobar los chancros y todo tipo de lesiones infectadas. El autor las divide en benignas y malignas basándose en su aspecto o en la posible infección que presentan. Las benignas curan y se extirpan con mayor facilidad. En cuanto a las lesiones malignas recomienda extirpar por completo "lo ennegrecido y corrompido" y utilizar el cauterio lunar (Figura 3), adaptado a la circunferencia del pene, si se produce una hemorragia. En el caso que se produzca una pérdida parcial o total del glande el autor 
da una gran importancia a la tutorización de la uretra para su correcta curación.

\section{4) Adherencias balanoprepuciales.}

Las adherencias se pueden producir en pacientes que tienen un prepucio sano y en los que presentan patología inflamatoria o infecciosa (herida o absceso). La solución de este proceso depende de la gravedad del cuadro y de la firmeza de las adherencias utilizando en los casos más complicados una espátula. Recomienda la colocación de un paño húmedo y curas posteriores para que no se vuelva a pegar.

\section{5) Circuncisión.}

No define la fimosis en ninguna parte de este capítulo. Solamente en el enunciado nos indica que va a tratar un tipo de patología infantil "algo que los niños no tienen bien", sin dar posteriormente ningún dato sobre síntomas o signos de la exploración física.

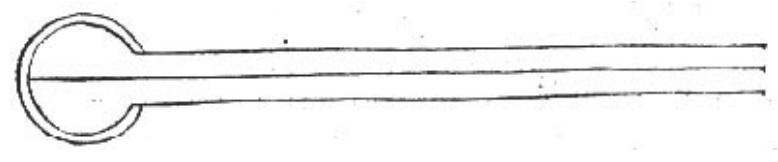

FIGURA 5. Siringa

Tomado de Teodoro Prisciano "Albucasis Chirurgicorum omnium Primarii, Lib tres". 1532 por loannem Schottum. Biblioteca digital Dioscórides. Biblioteca Marqués de Valdecilla. Universidad Complutense. Madrid.

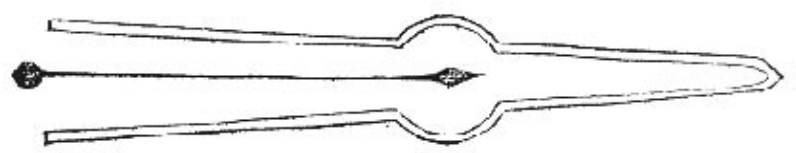

FIGURA 6. Clepshydra

Tomado de Teodoro Prisciano "Albucasis Chirurgicorum omnium Primarii, Lib tres". 1532 por loannem Schottum.

Biblioteca digital Dioscórides. Biblioteca Marqués de Valdecilla. Universidad Complutense. Madrid.

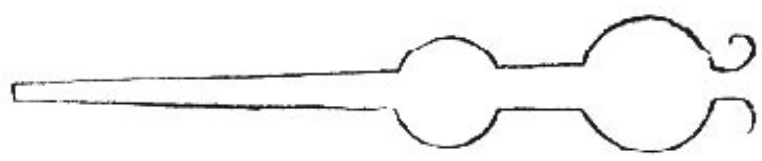

FIGURA 7. Clisteris subtilis

Tomado de Teodoro Prisciano "Albucasis Chirurgicorum omnium Primarii, Lib tres". 1532 por loannem Schottum. Biblioteca digital Dioscórides. Biblioteca Marqués de Valdecilla. Universidad Complutense. Madrid.
Abulcasis cita en primer lugar algunas formas de practicar la circuncisión mediante la utilización de cuchillas y flebotomos para realizar el corte en el prepucio indicando los problemas que se pueden plantear con estas técnicas como son la sección asimétrica $o$ irregular. También se refiere al "alfecati" que es una especie de huso hueco en el centro con el que se intentaba practicar una sección regular y circular, introduciendo dentro del mismo el pene, aunque con el peligro de lesionar el glande (31).

Pero la innovación que introduce el autor es la utilización de unas tijeras para que el corte sea simétrico en las dos caras del prepucio, la externa y la interna, colocando previamente a la sección dos ligaduras en el prepucio. No realiza sutura de la incisión practicada.

El dibujo de la tijera (Figura 4) que aparece en este capítulo es de baja calidad y de difícil interpretación.

\section{6) Retención de orina.}

No define la retención de orina aunque si enumera las causas que la producen: un cálculo, coágulos de sangre, pus o un tumor carnoso.

En esta edición se llama "siringa" (Figura 5) al instrumento con el que se procede a cateterizar la vejiga. En primer lugar realiza la descripción de este tipo de sonda que debe ser de plata, ligera y hueca con una longitud de palmo y media y con un embudo en el extremo colocando en su interior un hilo de lana.

Podemos deducir que Abulcasis da una gran importancia a la lubricación recomendando no solo aplicar materias lubricantes como aceite, manteca o clara de huevo en la sonda, sino también en el pene y en la vejiga (hipogastrio).

La técnica utilizada no difiere de descripciones anteriores. En primer lugar introduce la sonda hasta llegar a la región bulbar y después debe dirigirla hacia el ombligo con objeto de conducirla por la curva de la uretra bulbar debiendo empujar hasta llegar al interior de la vejiga. Posteriormente se saca el hilo de lana para que salga la orina.

\section{7) Forma de instilar líquidos en la vejiga.}

Abulcasis describe por primera vez en la historia de la cirugía un método para introducir líquidos en la vejiga (22). Esta actuación en la edición con la que trabajamos, recibe el nombre de "clisterización" y lo practica con dos instrumentos sin tener que introducir una sonda en vejiga (9): 
- La "clepshydra" es un modelo de jeringa cuyo dibujo en esta edición es difícil de interpretar (Figura 6). Se trata de un tubo hueco y un émbolo con el que se proyecta el líquido colocado en su interior. El término clepshydra se refiere a un reloj de agua que consiste en un recipiente que deja gotear agua por su extremo inferior. En este capítulo Abulcasis cita a la"clepshydra" como un instrumento que era utilizado para arrojar nafta ardiendo, el llamado "fuego griego", en las batallas navales.

- El "clisteris subtilis" (Figura 7) es un recipiente hecho de plata o auricalco (bronce, latón) (17) y utilizado para aplicar enemas pero con la salida más estrecha con el objeto que se pueda introducir por el meato uretral y diseñado para que se le pueda adaptar, de la mejor manera posible entre dos esferas, una bolsa hecha con vejiga de carnero o pergamino en la que se pone el líquido para la instilación (Figura 8) (20).

\section{8) Litiasis vesical en el varón.}

La litiasis vesical es la patología que Abulcasis trata con más extensión centrándose en el tratamiento quirúrgico de la misma. La operación de la talla vesical para la extracción de cálculos es una de las intervenciones más antiguas y temidas en la historia de la cirugía. Descrita anteriormente por Celso y Pablo de Egina, Abulcasis toma nota de diferentes detalles pero hace algunas innovaciones para tratar esta patología con mayor seguridad y menor morbilidad. El autor hace los siguientes apartados para practicar la que llama "operación con la mano y corte":

- Sintomatología. El autor cita los síntomas de manera resumida: emisión de arenillas blancas y de orina como el agua, el paciente se mira y rasca y toca el pene, tiene erecciones y se prolapsa el ano.

- Preparación del enfermo. Recomienda movimientos encaminados a dirigir la piedra al cuello vesical y lavativas que vacíen de heces el recto, con objeto de facilitar la localización del cálculo mediante el tacto rectal.

- Colocación del enfermo para realizar la cirugía. Este apartado lo describe con brevedad pero podemos deducir que un ayudante sujeta al paciente en posición de litotomía forzada para que "la vejiga se incline hacia abajo".

- Localización del cálculo. Se realiza mediante la combinación de palpación abdominal y tacto rectal, recomendando dirigir la piedra hacia el cuello vesical y comenzar la intervención inmediatamente en el caso de que se localice. Este apartado es importante ya que no era raro encontrar cálculos de gran tama- ño debido entre otros motivos a la larga evolución de esta enfermedad y al miedo a realizar la operación de la talla vesical por la elevada morbimortalidad que presentaba, con lo que se retrasaba la intervención y se daba opción en muchas ocasiones a un crecimiento excesivo de los cálculos.

- Incisión. La realiza en la región perineal con un bisturí llamado "spatumile incidens" comenzando a practicarla entre el ano y los testículos pero no en la línea media sino dirigiéndose hacia la nalga izquierda de manera transversal. Recomienda hacerla más amplia en la piel que en tejidos más profundos. No debe ser mayor que la piedra y hay que evitar el hacerla de gran tamaño por el riesgo de que se pueda producir una fístula vésicocutanea (24).

- Extracción de la piedra. La realiza mediante presión con el dedo al realizar el tacto rectal o ayudándose con unas tenazas o un instrumento "delgado y curvo" que introduce por la incisión. En el caso de que la piedra sea grande debe ser fragmentada mediante unas tenazas y proceder posteriormente a su extracción. La litotricia vesical es una aportación innovadora de Abulcasis a esta intervención.

- Instrumental. La incisión la realiza con un bisturí que el autor de la edición con la que trabajamos llama "spatumile incidens" (Figura 9), adjuntando un grabado de buena calidad. Presenta la innovación de tener los dos bordes cortantes (16). Las tenazas y el "instrumento delgado y curvo" no son descritos gráficamente en este libro.

- Cura postoperatoria. La realiza introduciendo en la herida thure, áloe y sangre de dragón realizando una fuerte compresión y colocando un apósito con aceite $\circ$ vino $o$ aceite de rosas y agua fría como antiinflamatorio. Posteriormente utiliza ungüento dyafinicon y basilicón.

- El "thure" es el incienso (3) y se utilizaba para curar las heridas. Se obtiene de la resina del olíbano (Boswellia thurifera).

- El áloe y la sangre de dragón son plantas medicinales con propiedades cicatrizantes y antisépticas.

- El ungüento basilicón está compuesto por cera, pez y colofonia y su aplicación tiene propiedades antiinflamatorias (17).

- El ungüento "dyafinicon" se pensaba que "purgaba los humores de la flema y de la cólera" (19).

- Manejo de las complicaciones. Se refiere fundamentalmente a dos situaciones: 
- Infección de la herida quirúrgica

- Hematuria y formación de coágulos en vejiga.

Aporta su experiencia en la resolución de estos dos graves problemas que suponían una complicación muy importante en la evolución postoperatoria de la intervención de la talla vesical. La formación de coágulos dentro de la vejiga hace obligada su extracción con objeto de prevenir la infección de los mismos y un estado séptico subsiguiente que llama "la corrupción y putrefacción de la vejiga".

\section{9) Litiasis vesical en la mujer.}

Es la primera vez que se cita la intervención de la talla, según Kurt Sprengel, para resolver una litiasis vesical en la mujer $(15,18)$. Para el autor esta patología es rara y su tratamiento presenta una serie de obstáculos que se derivan de la costumbre del pueblo árabe de que los genitales de la mujer sólo

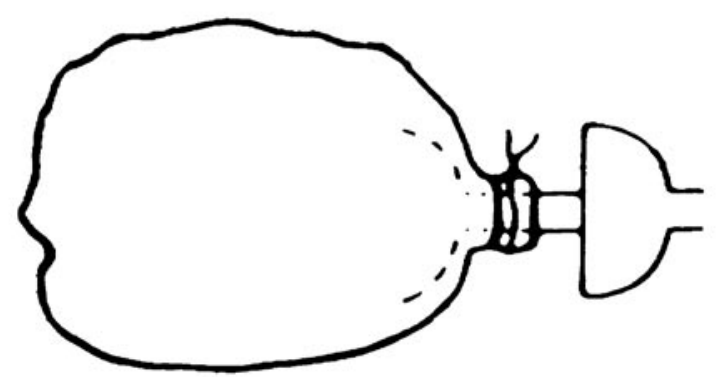

FIGURA 8. Clisteris subtilis a la que se adapta una vejiga de carnero o un pergamino. Tomada de: Maganto Pavón, E. y cols. en "Hitos de la Urología".

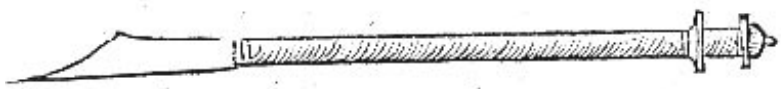

FIGURA 9. Spatumile incidens Tomado de Teodoro Prisciano "Albucasis Chirurgicorum omnium Primarii, Lib tres". 1532 por loannem Schottum. Biblioteca digital Dioscórides. Biblioteca Marqués de Valdecilla. Universidad Complutense. Madrid.

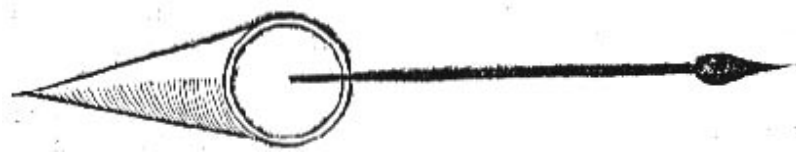

FIGURA 10. Perforatorium

Tomado de Teodoro Prisciano "Albucasis Chirurgicorum omnium Primarii, Lib tres". 1532 por loannem Schottum. Biblioteca digital Dioscórides. Biblioteca Marqués de Valdecilla. Universidad Complutense. Madrid. podían ser vistos por otra mujer. Por lo citado anteriormente era posible encontrarse con los siguientes problemas:

- Que la mujer fuera virgen.

- Era difícil que una mujer se desnudara ante un médico.

- No había una mujer que conociera la intervención quirúrgica para solucionar este problema.

- Debido a la anatomía femenina, la distancia entre la piel y un cálculo es mayor en la mujer que en el hombre, "la lejanía del lugar", por lo que la incisión debe ser más profunda y era más arriesgado.

La técnica sigue los siguientes pasos:

- Recolocación del cálculo cerca del cuello vesical mediante el tacto rectal o vaginal combinado con la palpación abdominal.

- Incisión sobre la piedra entre el centro de la vulva y la parte inferior de la raíz del muslo, similar a la practicada en el hombre, adaptándola al tamaño de la piedra.

- Introducción de una sonda por la incisión para la localización correcta del cálculo

Si se presenta una hemorragia recomienda poner una molienda de vitriolo en la herida y esperar a que cese. En el caso de que ésta sea importante, como la secundaria a una sección arterial, se debe comprimir con cojines "de una manera apropiada".

\section{0) Litiasis uretral.}

Este apartado es el capítulo más interesante de la obra quirúrgica urológica de Abulcasis y posiblemente el más innovador. Por primera vez se describe la litotricia en una litiasis uretral enclavada.

La intervención la realiza de la siguiente manera. En primer lugar localiza y fija el cálculo en la uretra, seguidamente lo fragmenta mediante un instrumento que en la edición que estamos utilizando recibe el nombre de "perforatorium" (Figura 10). Se puede comprobar el diseño del mango que tiene por objeto adaptarse a la mano para poder hacer la mayor fuerza posible contra el cálculo que se aplica a través de un extremo de acero terminado en punta triangular. Una vez fragmentado se procede a su extracción.

En el caso de no peder practicar lo anterior realiza una uretrotomía externa para la extracción 
del cálculo previa colocación del mismo entre dos ligaduras.

\section{1) Hidrocele (hernia acuosa).}

Dentro de la patología testicular define los siguientes apartados:

- Hernia acuosa o hidrocele: Es la colección de líquido en la vaginal testicular "sisac" que contiene el testículo, tiene forma redondeada y algo alargada y no se palpa el testículo.

- Quiste de cordón o de epidídimo: Colección de líquido junto al testículo en un tejido "apropiado para él" y que parece que se trata de otro testículo.

Describe los diferentes colores del líquido y la punción del hidrocele mediante un trocar cuadrado para conocer las características del mismo, aunque esto no va a modificar su actitud terapéutica. La intervención quirúrgica sobre el hidrocele contempla tres aspectos:

a) Tratamiento quirúrgico convencional. Se dirige a vaciar de líquido la cavidad escrotal y a tratar de evitar la recidiva del hidrocele mediante la extirpación de la mayor parte posible de la vaginal testicular. Como podemos comprobar recomienda muchos gestos quirúrgicos que practicamos en la actualidad como son:

- Al realizar la incisión no perforar la vaginal testicular (sisac blanco) "guárdate de cortarlo" ya que dificultaría el paso siguiente.

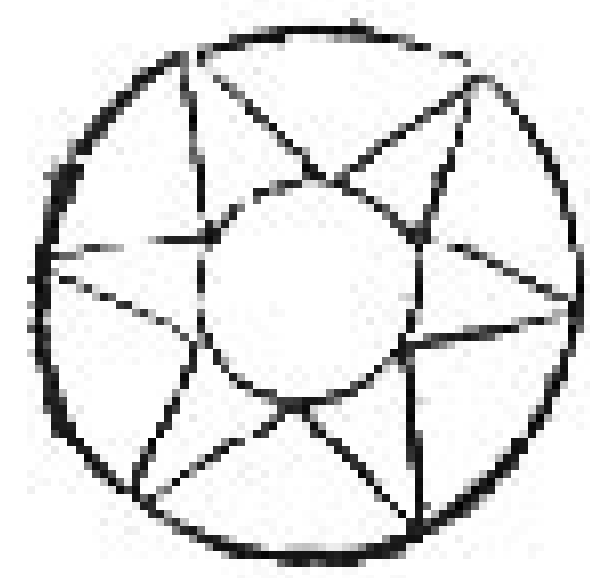

FIGURA 1 1. Suspensorio

Tomado de Teodoro Prisciano "Albucasis Chirurgicorum omnium Primarii, Lib tres". 1532 por loannem Schottum. Biblioteca digital Dioscórides. Biblioteca Marqués de Valdecilla. Universidad Complutense. Madrid.
- Despegar la piel escrotal de la vaginal testicular antes de vaciar el hidrocele "desollamiento de la piel".

- Extirpar la mayor parte posible de la vaginal.

- Sutura de la herida quirúrgica.

Para el postoperatorio recomienda la colocación de un suspensorio preparado con unos paños rellenos de lana cardada que ata a un cinturón mediante seis tirantes. En la edición con la que trabajamos el dibujo del suspensorio es poco afortunado $y_{\text {, }}$ como en casos anteriores, de difícil interpretación. Aparece como una estrella de seis puntas que representan cada uno de los tirantes que se atan al cinturón representado por la circunferencia que rodea la estrella (Figura 11).

b) Tratamiento mediante el cauterio. Abulcasis también se refiere a la cura con cauterio siguiendo los mismos pasos que con la cirugía convencional. Para ello utiliza un instrumento en cuyo extremo tiene forma de letra griega sin decir en la edición que utilizamos de cual de ellas se trata. En otra edición aparece la letra griega tau T (Figura 12) (31).

c) Punción evacuadora. La considera un tratamiento paliativo ya que según el autor la recidiva se producirá en seis meses. Se practica con un bisturí ayudándose con una pluma a modo de sonda con el objeto de vaciar por completa la cavidad de líquido. También recomienda hacer la incisión con el instrumento recomendado por el autor en el Capítulo LIII para vaciar el líquido de los hidrópicos cuyo dibujo se encuentra en la página 197 de la presente edición (Figura 13).

\section{2) Tumores testiculares (hernia larga carnosa).}

En este apartado el autor se refiere a los tumores carnosos y a las inflamaciones testiculares. La califica de una patología grave y el tratamiento que recomienda es la abstención quirúrgica ya que la intervención era considerada de riesgo. Recomienda la orquidectomía si la tumoración no se puede separar del testículo, explicando los pasos a seguir con una gran claridad:

- "Extrae el mismo (testículo) junto con el sisac blanco".

- "Libera el suspensorio" (cordón espermático).

- "Ata los vasos".

- "Corta el suspensorio".

\section{3) Varicocele (rama larga que existe con la vid).}

Compara el varicocele a un racimo de uvas y lo asocia con la producción de una atrofia testicular (ablandamiento del testículo). 
El tratamiento quirúrgico va encaminado a disecar los vasos varicosos haciendo una ligadura en la parte más alta del varicocele y otra en la parte más baja procediendo a abrir longitudinalmente los vasos dilatados para que salga la sangre contenida en los mismos que la llama "turbia humedad corrompida".

Como podemos comprobar practica una variante de la ligadura parcial del plexo pampiniforme, técnica que no se practica en la actualidad pero que está descrita en los tratados de cirugía urológica.

\section{4) Reblandecimiento de la piel del escroto.}

En este capítulo no cita una patología clara ni determinada. No sabemos con seguridad a qué enfermedad se refiere, tal vez a situaciones de linfedema que causan un deterioro estético del escroto. La solución quirúrgica es la extirpación de la piel que se encuentre en mal estado.

\section{5) La castración.}

La castración está prohibida por la ley musulmana pero no obstante en los capítulos LXII, LXIII y LXIV la describe y explica su realización para cuando exista una patología que requiera este tratamiento. En el presente capítulo, así lo recuerda el autor que era un ferviente musulmán, deja una puerta abierta a la práctica de la misma en caso de necesidad. Fuera de la patología testicular no tiene aplicación en el hombre.

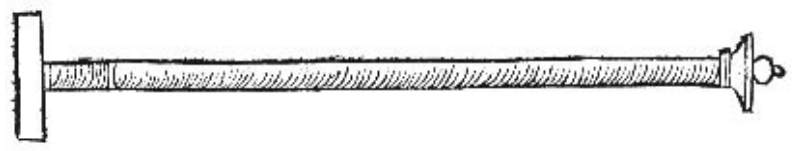

FIGURA 12. Cauterio en T Tomado de Teodoro Prisciano "Albucasis Chirurgicorum omnium Primarii, Lib tres". 1532 por loannem Schottum. Biblioteca digital Dioscórides. Biblioteca Marqués de Valdecilla. Universidad Complutense. Madrid.

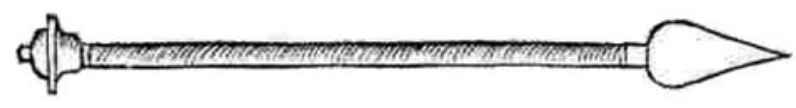

FIGURA 13. Instrumento para hacer la punción del hidrocele.

Tomado de Teodoro Prisciano "Albucasis Chirurgicorum omnium Primarii, Lib tres". 1532 por loannem Schottum. Biblioteca digital Dioscórides. Biblioteca Marqués de Valdecilla. Universidad Complutense. Madrid.
16) Hermafroditismo y su tratamiento quirúrgico.

No define lo que es un hermafrodita pero si apunta la existencia de ambos tipos de genitales externos en los dos sexos: presencia de una vulva junto a los testículos en el varón y de un pene y testículos en la vulva de la mujer. El tratamiento es la extirpación de las "carnes añadidas" salvo en el caso del hombre en que exista salida de orina por los genitales femeninos.

\section{CONCLUSIONES}

- Abulcasis es el primer médico nacido en la Península lbérica que escribe un tratado quirúrgico en el que describe algunas intervenciones urológicas.

- Se basa en las ideas quirúrgicas de autores clásicos.

- Es un gran entusiasta en la utilización del cauterio.

- Describe por primera vez la talla vesical en la mujer.

- Describe por primera vez la litotricia vesical.

- Describe por primera vez la litotricia uretral.

- Por primera vez se realiza una descripción gráfica de instrumental quirúrgico.

- Es el inventor de múltiples instrumentos quirúrgicos. Dentro del instrumental urológico diseña el perforatorium para practicar la litotricia uretral.

\section{AGRADECIMIENTOS}

El texto latino ha sido traducido por María Jesús de Irízar, Licenciada en Filología Clásica por la Universidad Complutense de Madrid, representando una ayuda inestimable para la correcta interpretación de esta obra.

\section{BIBLIOGRAFÍA y LECTURAS RECOMENDADAS (*lectura de interés $y^{* *}$ lectura fundamental)}

1. ABDEL-HALIM, R.E.: "Lithotripsy: A historical review", Tercer Congreso de la Sociedad Internacional de Endoscopia Urológica junto con la W.H.O. Agosto 1.984. Karlsruhe. Editado por Werner Steinbruck, pag. 474-476, 1985. 
*2. ABDEL-HALIM R.E.; ALTWAIJIRI, A.S.; ELFAQIH, S.R. y cols.: "Extraction of urinary stone as described by Abul-qasim Khalaf Ibn Abbas Alzahrawi (Albucasis) (325-404 H, 930-1013 AD). A translation of original text and a commentary". Saudi Med J., 24: 1283, 2003.

*3. KAADAN, A.N.: "Abulcasis and extraction of Bladder stone". Journal of the International Society for the History of Islamic Medicine (JISHIM), 3: $28,2004$.

4. AL-GHAZAL, S.K.: "Al Zahrawi (Albucasis)- a light in the dark middle ages in Europe" Journal of the International Society for the History of Islamic Medicine (JISHIM), 1: 37, 2003.

5. ARJONA CASTRO, A.: "Albucasis y la gota de Almanzor". Boletín Informativo del Colegio Oficial de Médicos de la Provincia de Córdoba, Córdoba, 118, Mayo, 1985.

6. ARVIDE CAMBRA, L.M.: "Un tratado de polvos medicinales en Al-Zahrawi". Universidad de Almería, 1994.

7. ARVIDE CAMBRA, L.M.: "Tratado de pastillas medicinales según Abulcasis". Universidad de Almería, 1996.

8. CAMPBELL, D.: "Arabian medicine and its influence on the middle ages". Londres, 1926.

9. CIFUENTES DÍAZ, P.: "Bosquejo histórico de la Urología en España anterior al siglo XIX”. Madrid, 1945.

*10. DOGNÉE, E.: "La higiene de Albucasis". Publicaciones de la Academia de Ciencias Médicas de Córdoba, Córdoba, 1925.

11. FALAGAS, M.E.: "Arab science in the golden age (750-1.258 C.E.) and today". FASEB. J., 20: 1581, 2006.

12. GOYANES CAPDEVILA, J.: "La Cirugía de Abulcasis". Gaceta Médica Española XXVII, 4: 601, 1953.

13. GOYANES CAPDEVILA, J.: "Comentarios al texto de la Cirugía de Abulcasis". Gaceta Médica Española XXVIII, 6: 201, 1954.

14. HAMARNEH, S.: "Drawings and pharmacy in al-Zahrawi's 10th-Century surgical treatise". Washington, 1961.

15. HERNÁNDEZ MOREJÓN, A.: "Historia bibliográfica de la Medicina española". New York, 1967.

16. KAADAN, A.N.: "Abulcasis and extraction of bladder stone". Journal of the International Socie- ty for the History of Islamic Medicine (JISHIM), 3: 28, 2004.

17. LAROUSSE LIBRAIRIE.: "Nueva Enciclopedia Larousse". $3^{\mathrm{a}}$ ed. Barcelona, 1984.

*18. LECLERC, L.: "Histoire de la Médecine arabe". Livre III, 448-457, New York, 1971.

19. LUQUE SÁNCHEZ, M.E.; CAÑADILLAS DE LA FUENTE, M.A.; CRESPO MUÑOZ, F.J.: "Estudio de la botica de la Alhambra en el siglo XVI". Ars. Pharm., 47: 275, 2006.

20. MAGANTO PAVÓN, E.; CLEMENTE RAMOS, L.; CARRERA PUERTA, C.: "Hitos en la Historia de la Urología". Tomo I, Ediciones Pulso, S.A. Barcelona, 1999.

*21. MAGANTO PAVÓN, E.: "Historia Biográfica y Bibliográfica de la Urología Española". Edad Antigua y Medievo, Edicomplet. Madrid, 2000.

22. MAHMOOD AL-HAJ KASIM, M.: "Investigation and empting instruments of arab and Moslem surgeons". Journal of the International Society for the History of Islamic Medicine (JISHIM), 3: 2, 2004.

*23. MORENO RESINA, J.M.: "La cirugía de Abulcasis" Tesis doctoral. Universidad de Murcia, 2005.

24. MURPHY, L.: "The History of Urology". Springfield, 1972.

25. OTERO TEJERO, I.J.; GOLBANO ABLANQUE, J.M.; SERRANO PASCUAL, A. y cols.: "La patología uretral congénita en la obra de Abulcasis". Póster LXVI Congreso Nacional de Urología, Granada, 2001.

**26. PRISCIANO, T.: "Albucasis Chirurgicorum Omnium Primarii. Liber Tres". Estrasburgo, 1532.

27. RIERA, J.: "Historia de la Cirugía". Madrid, 1993.

28. RIERA, J.: "El capítulo urológico en la obra quirúrgica de Abulcasis". Actas del II Congreso Español de Historia de la Medicina, Salamanca, 1: 85, 1966.

29. SÁNCHEZ GRANJEL, L.: "Retablo histórico de la urología española". Universidad de Salamanca, 1986.

30. SCHIPPERGES, H.: "La medicina árabe en el medievo latino". Real Academia de Bellas Artes y Ciencias Históricas de Toledo, 1989.

**31. SPINK, M.S.; LEWIS, G.L.: "Albucasis on Surgery and Instruments. A definitive edition of the arabic text with english translation and commentary”. London, 1973. 